\title{
Extracellular production of recombinant sus scrofa trefoil factor 3 by Brevibacillus choshinensis
}

\author{
HE-PING LI $^{1 *}$, CHUN-MEI XU $^{1 *}$, BING-YAN WEN $^{1 *}$, AN-QI LI $^{2}$, GUANG-MING ZHA $^{1}$, XIANG-YANG JIN ${ }^{1}$, \\ YUN-ZE ZHAO $^{1}$, LU-PING FENG ${ }^{1}$, YE-DONG CAO ${ }^{1}$, GUO-YU YANG ${ }^{1}$, YUE-YING WANG ${ }^{1}$ and KAI ZHONG $^{1}$ \\ ${ }^{1}$ Key Laboratory of Animal Biochemistry and Nutrition, Ministry of Agriculture, Henan Agricultural University, Zhengzhou, \\ Henan 450002; ${ }^{2}$ Kansas International College, Zhengzhou Sias University, Xinzheng, Henan 451100, P.R. China
}

Received June 27, 2019; Accepted December 12, 2019

DOI: $10.3892 /$ etm.2020.8477

\begin{abstract}
Trefoil factor 3 (TFF3) is involved in cell adhesion, motility and apoptosis, regulates mucosal immunity and maintains the functional integrity of intestinal epithelia. The upregulation of TFF3 expression in the weaning rat intestine attracted our interest. The present study hypothesized that TFF3 may serve a role in preventing diarrhea in weaning piglets, which is an important consideration in the pig farming industry. Previous recombinant TFF3 protein expression yields obtained from Escherichia coli were too low and the bioactivity of the protein was poor. Hence, this expression system was unsuitable for industrial applications. The present study explored the production of recombinant sus scrofa TFF3 in a Brevibacillus choshinensis (B. choshinensis) expression system, aiming to enhance the expression level of bioactive protein. To achieve this, the sus scrofa TFF3-encoding gene fragment was fused into an E. coli-Brevibacillus shuttle vector pNCMO2. High levels of TFF3 $(30 \mathrm{mg} / \mathrm{l})$ were produced and secreted into the $B$. choshinensis culture medium in soluble form with a molecular mass of $13.6 \mathrm{kDa}$ and high immunoreactivity in western blotting. Thus, Brevibacillus may be used to produce useful mucosal factors for biochemical analyses and mucosal protection, and in industrial applications to produce novel inhibitors of diarrhea.
\end{abstract}

\section{Introduction}

The family of Trefoil factor (TFF)-peptides, previously known as P-domain peptides, consists of 3 members (TFF1, 2 and 3) (1).

Correspondence to: Professor Yue-Ying Wang or Professor Kai Zhong, Key Laboratory of Animal Biochemistry and Nutrition, Ministry of Agriculture, Henan Agricultural University, 63 Nongye Road, Zhengzhou, Henan 450002, P.R. China

E-mail: wangyueying2008@126.com

E-mail: zhongkai@henau.edu.cn

*Contributed equally

Key words: protein expression, trefoil factor 3, pNCMO2, Brevibacillus choshinensis
TFF-peptides are small proteins secreted by a number of mucosal epithelial cells (2). TFFs possess a common trefoil domain structural motif, forming characteristic disulfide bonds through six conserved cysteine residues, and are highly gastric acid and protease-resistant (2). TFFs help maintain mucosal barrier integrity by protecting the gastrointestinal (GI) mucosa against injury and improving repair following injury (3-5). The pit cells in gastric mucosa primarily secret TFF1, whereas TFF2 is synthesized by the neck cells of the gastric mucosa and the Brunner's glands of the duodenal submucosa (6). The distribution of TFF1 and TFF2 is confined to the proximal GI tract, whereas TFF3 is produced by the goblet cells and secreted abundantly throughout the intestinal tract (3). TFF3 protects and repairs the gastrointestinal mucosa, maintains the tight junction barrier integrity and restores normal intestinal permeability during inflammatory bowel disease pathogenesis (7). TFF3 is also present in the neural lobe of the porcine pituitary gland $(8,9)$.

The GI tract acts as a barrier against bacteria and toxins preventing digestive disorders. Weaning is an important process during development of the pig (10). The structure and function of the digestive system of a growing pig begins immediately to change when weaning begins (10). Weaning involves extensive exposure to novel antigens, improving the redistribution of the microbial community in the small intestine (10). Piglets are susceptible to disease after weaning. When the GI mucosal barrier function is impaired, the ecological balance among gut microbes is destroyed leading to enterogenous infection, resulting in diarrhea (11). Diarrheal piglets are of significant concern to the pig industry (10). Previous studies have revealed that TFFs can maintain the integrity of the intestinal epithelia in mice, heal wounds in humans and participate in cell adhesion, motility and apoptosis in vitro (12-17). TFFs are involved in mucosal immune modulation by regulating leukocyte recruitment and repairing tissue to ensure healthy mucosal surfaces (18). In addition, the level of TFF3 expression is increased in the intestine of weaning rats (19). Thus, the present study hypothesized that TFF3 may serve an important role in preventing diarrhea in weaning piglets.

In previous studies, TFF3 has been expressed in Escherichia coli in an intracellular and soluble form (20-22). However, this method of production is not ideal because of the low yield and bioactivity of the produced TFF3 (20-22). 
The present study developed a recombinant expression system for sus scrofa TFF3 fused with a 6xhis-tag in a strain of B. choshinensis. B. choshinensis is a Gram-positive bacterium, which has the excellent advantage of secreting various extracellular proteins with high efficiency (23). The spore forming ability of $B$. choshinensis has been removed by genetic engineering. In addition, this expression system is very powerful for producing proteins with native structures, even when they contain disulfide bonds (23). pNCMO2 is a B. choshinensis- $E$. coli shuttle vector (24). The pNCMO2 vector includes the P2 promoter, which drives the transcription of cell wall protein but has no role in E. coli (24). The objective of the present study was to explore the production of sus scrofa TFF3 by $B$. choshinensis in vitro.

\section{Materials and methods}

Bacteria, plasmids and media. B. choshinensis strain HB116 (Takara Bio, Inc.) was used in this study. E. coli DH5a competent cells (Sangon Biotech Co, Ltd.) were used for DNA manipulation. pNCMO2 (Takara Bio, Inc.) and pMD19-T (Takara Bio, Inc.) were used as the vector and subcloning plasmid, respectively. Milk-Tween (MT) medium containing $2 \%$ yeast extract, $10 \%$ glucose, $10 \%$ polypeptone, $5 \%$ meat extract, $0.01 \% \mathrm{FeSO}_{4} \cdot 7 \mathrm{H}_{2} \mathrm{O}, 0.001 \% \mathrm{ZnSO}_{4} \cdot 6 \mathrm{H}_{2} \mathrm{O}$ and $0.01 \%$ $\mathrm{MnSO}_{4} \cdot 4 \mathrm{H}_{2} \mathrm{O}$ was used to culture strain HB116. E. coli $\mathrm{DH} 5 \mathrm{\alpha}$ cells were cultured in Luria Broth medium (Oxoid; Thermo Fisher Scientific, Inc.). $\mathrm{NaOH}$ was used to adjust the $\mathrm{pH}$ of all media to 7.0. Neomycin $(20 \mu \mathrm{g} / \mathrm{ml}$; Beijing Solarbio Science \& Technology Co, Ltd.) was added to the media used to culture bacteria containing pNCMO2 and derivatives.

RNA extraction and PCR. Total RNA from sus scrofa spleen tissue (preserved in our laboratory) was isolated using a TRIzol ${ }^{\circledR}$ reagent kit (Takara Bio, Inc.) according to the manufacturer's instructions. cDNA synthesis was performed using a PrimeScript Reverse Transcriptase kit (Takara Bio, Inc.). Primers for the sus scrofa TFF3 gene were designed using Primer v.5.0 software (Sangon Biotech. Co. Ltd.), according to the gene sequence in the GenBank database (accession no.NM_001243483).mRNA specific primers (Sangon Biotech Co, Ltd.) were: TFF3 forward, 5'-GCATGGAGGCCAGGA TGT-3' and reverse, 5'-CGGTTAGAAGGTGCATTCT-3'. The PCR program to amplify the TFF3 gene from cDNA was $95^{\circ} \mathrm{C}$ for $5 \mathrm{~min}$, followed by 35 cycles of $95^{\circ} \mathrm{C}$ for $30 \mathrm{sec}, 55^{\circ} \mathrm{C}$ for $20 \mathrm{sec}$ and $72^{\circ} \mathrm{C}$ for $30 \mathrm{sec}$ with a final extension step at $72^{\circ} \mathrm{C}$ for $10 \mathrm{~min}$. PCR products were separated by $2 \%$ agarose gel electrophoresis. Purified PCR fragments were retrieved using a PCR gel recovery kit (Takara Bio Inc.). Bands were visualized using the GelDoc $\mathrm{XR}^{+}$(Bio-Rad Laboratories, Inc.) gel imaging system through nucleic acid staining. Densitometric analysis was performed using Gel-Pro Analyzer 4.0 software (Media Cybernetics, Inc.).

Construction of pNCMO2-TFF3-6xhis. According to the manufacturer's instructions of the pMD19-T vector kit (Takara Bio, Inc.), the TFF3-encoding gene and 6xhis tag were linked to the $\mathrm{T}$ vector to generate pMD19-T-TFF3-6xhis and transformed into E. coli DH5 $\alpha$. Positive clones were identified by colony PCR, where a single bacterium is used as a template, and can quickly identify whether the colony is a positive colony with the target gene. Plasmids extracted from the positive clones were sequenced by Takara Bio, Inc. The primers were forward, 5'-GCgtcgacATGGAGGCCA GGATGT-3' and reverse, 5'-CGGggtaccTTAGTGATGTGA TGGTGATGGAAGGTGCATTCT-3'; lower-case letters denote the enzyme restriction sites for SalI and KpnI and the underlined bases denote the nucleotide sequence encoding the 6xhis tag. To construct the sus scrofa TFF3 expression vector pNCMO2-TFF3-6xhis, the fused TFF3-6xhis combined fragment from pMD19-T-TFF3-6xhis was amplified by PCR. The following thermocycling conditions were used for the PCR: initial denaturation at $95^{\circ} \mathrm{C}$ for $10 \mathrm{~min}$; followed by 35 cycles of $95^{\circ} \mathrm{C}$ for $30 \mathrm{sec}, 56^{\circ} \mathrm{C}$ for $30 \mathrm{sec}$ and $72^{\circ} \mathrm{C}$ for $30 \mathrm{sec}$; and a final extension $72^{\circ} \mathrm{C}$ for $10 \mathrm{~min}$. Digestion with SalI and $K p n I$ followed by ligation at $16^{\circ} \mathrm{C}$ overnight was used to clone the amplified fragment into the pNCMO2 vector to generate pNCMO2-TFF3-6xhis. DNA sequencing was performed to confirm the cloned DNA sequence (Takara Bio, Inc.).

B. choshinensis transfection. Competent cells of B. choshinensis HB116 were prepared by inoculating $1 \mathrm{ml}$ bacterial solution in $100 \mathrm{ml}$ MT medium (Shanghai Kemin Biotechnology Ltd.), which were then cultured at $37^{\circ} \mathrm{C}$ for $18 \mathrm{~h}$. Bacteria were then collected by centrifugation at $4,000 \mathrm{x} \mathrm{g}$ for $5 \mathrm{~min}$ at room temperature, and suspended in $50 \mathrm{mmol} / 1$ Tris- $\mathrm{HCl}$ ( $\mathrm{pH}=8.5$; Beijing Dingguo Changsheng Biotechnology Co, Ltd.). Bacteria were then transfected with vector DNA using electrophoretic transfer according to the manufacturer's instructions (Takara Bio, Inc.). The empty pNCMO2 vector was transfected as a negative control. A MicroPulser (Bio-Rad Laboratories, Inc.) was used with the Ec2 program.

Protein expression of recombinant sus scrofa TFF3. B. choshinensis containing pNCMO2-TFF3-6xhis or pNCMO2 was cultured in liquid MT medium containing $20 \mathrm{mg} / 1$ neomycin at $30^{\circ} \mathrm{C}$ for $60 \mathrm{~h}$. Once the bacteria reached the logarithmic growth period at $37^{\circ} \mathrm{C}$, isopropyl $\beta$-D-1-thiogalactopyranoside (final concentration $1 \mathrm{mmol} / \mathrm{l}$ ) was added to the bacteria. Following induction, the collected bacteria were disintegrated by ultrasound (JY88-II Ultrasonic Cell Disruptor; Bio-Equip) and lysed in PBS ( $\mathrm{pH}=7.4)$. The bacterial precipitate and supernatant were subsequently used in SDS-PAGE. Supernatants and cells were separated by centrifugation at $12,000 \mathrm{x} \mathrm{g}$ for $20 \mathrm{~min}$ at $4^{\circ} \mathrm{C}$. Proteins were detected by reducing $14 \%$ SDS-PAGE stained with Coomassie Brilliant Blue, and the amount of secreted proteins was evaluated. Gel-Pro Analyzer 4.0 software (Media Cybernetics, Inc.) was used to scan and measure the density of bands on gels to evaluate the expression level of the target protein. For western blotting, protein samples were boiled in SDS/b-mercaptoethanol loading buffer (192 mM glycine and 25 mM Tris; pH 8.3; Beijing Dingguo Changsheng Biotechnology Co, Ltd.) and subsequently electrophoresed. A total of $10 \mu \mathrm{g}$ protein/lane was separated via SDS-PAGE on a $14 \%$ gel. Proteins in the gel were transferred onto PVDF membranes (Merck KGaA) by electrophoretic transfer. Next, the membrane was blocked for $1 \mathrm{~h}$ in $5 \%$ skim milk at room temperature. TBST [20 mM Tris ( $\mathrm{pH} 8.0$ ), $200 \mathrm{mM} \mathrm{NaCl}$ and $0.1 \%$ Tween 20 ] was used to dilute the mouse anti-6xhis-tag 
monoclonal primary antibody (Abcam; cat. no. ab18184; $1: 2,000)$. The membrane was incubated overnight with the primary antibody at $4^{\circ} \mathrm{C}$ and washed with TBST three times for $15 \mathrm{~min}$. Horseradish peroxide-conjugated secondary antibody (Abcam; goat-mouse IgG; cat. no. ab150113) was diluted 1:3,000 with TBST and incubated with the membrane for $1 \mathrm{~h}$ at room temperature. Washing with TBST was subsequently performed three times for $15 \mathrm{~min}$. ECL development methods and X-ray film exposure were used to visualize the bands (Merck KGaA).

\section{Results}

Cloning of the TFF3 gene. The coding DNA sequence (CDS) region of the sus scrofa TFF3 gene (NM_001243483) was searched for in the NCBI database. The length of the TFF3 CDS is 243 bp (25). In the present study, the TFF3 CDS was cloned and a $\sim 250$ bp PCR product was obtained (Fig. 1). Sequencing confirmed the nucleotide sequence and length of the target band (data not shown).

Verification of pNCMO2-TFF3-6xhis protein fragment. pNCMO2-TFF3-6xhis was generated by cloning the TFF3 CDS into pNCMO2. pMD19-T-TFF3-6xhis was verified by a restriction enzyme digest, which produced two fragments of the expected sizes 273 and 2,692 bp (Fig. 2). pNCMO2-TFF3-6xhis was verified by PCR using the total extracted DNA as the template (Fig. 3).

Verification of recombinant $B$. choshinensis. pNCMO2TFF-6x his was electroporated into the $B$. choshinensis strain HB116. The resulting strain HB116-pNCMO2-TFF3-6xhis was verified by double enzyme digestion using extracted plasmid DNA as the template, SalI and KpnI were used as restriction enzymes to digest plasmid. The size of target band and plasmid were 273 and 5,200 bp, respectively (Fig. 4).

Analysis of the recombinant fusion protein. Protein samples from the culture medium of HB116 cells, which were transformed with the pNCMO2-TFF3-6xhis plasmid, were detected using SDS-PAGE. A band with a molecular weight of $\sim 55 \mathrm{kDa}$ in the supernatant (lane 1; Fig. 5) and precipitate (lane 2; Fig. 5) of the lysate of positive control vector-amylase, while a recombinant protein with a molecular weight of $\sim 13 \mathrm{kDa}$ was secreted by the supernatant and precipitate of HB116-pNCMO2-TFF3-6xhis (lane 5 and 6, respectively; Fig. 5). In western blotting, no immunoreactive band was present in the negative control lane, in which vector-free bacterium were induced. However, bacteria transfected with pNCMO2-TFF3-6xhis plasmid after induction displayed high immunoreactivity for the his-tag antibody (Fig. 6).

\section{Discussion}

TFF3 is a small secreted peptide and potential cytokine involved in protecting the GI mucosa (3). TFF3 can alleviate injuries in the GI mucosa caused by numerous types of stimulus and quicken repair of damaged mucosa $(2,3,7)$. TFF3 enhances the integrity of the mucosal epithelial surface and promotes

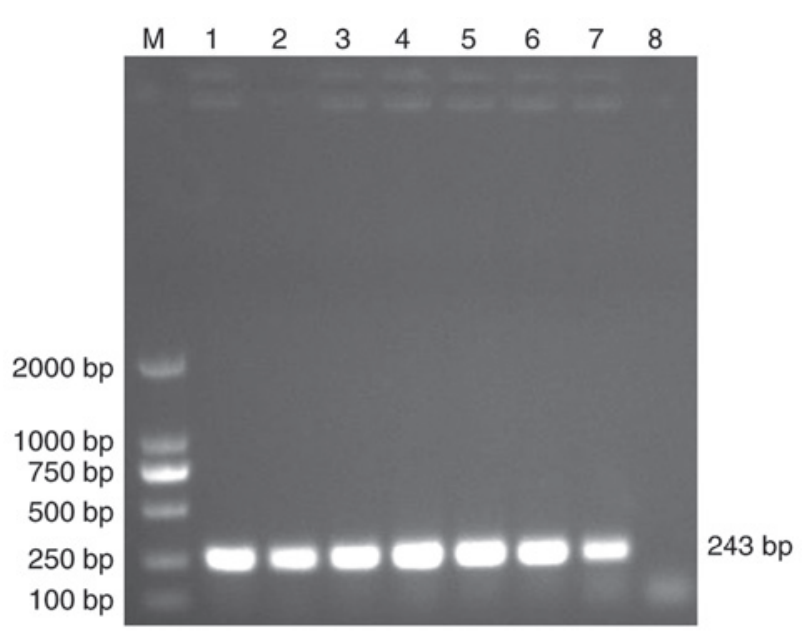

Figure 1. Electrophoretic analysis of amplified products for sus scrofa TFF3 gene. Lane 1-7, PCR products of TFF3 gene; lane 8, negative control, the PCR product without template DNA; M, DNA marker; TFF3, trefoil factor 3.

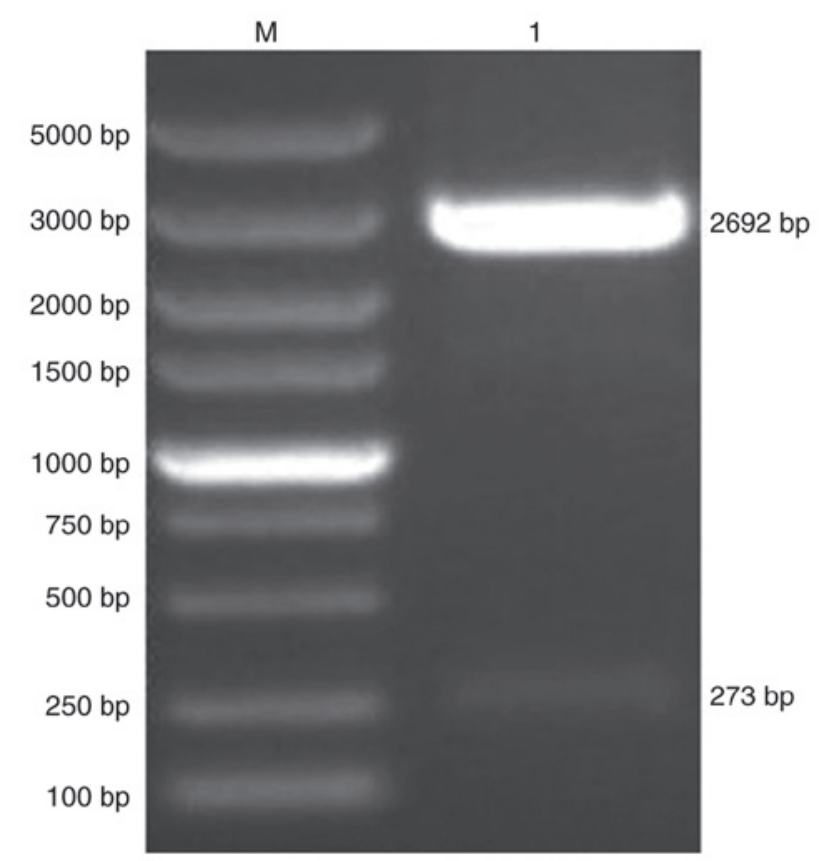

Figure 2. Identification of double enzyme digestion for pMD19-T-TFF3-6xhis plasmid. Lane 1, vector pMD19-T-TFF3-6xhis digested by restriction enzyme Sal I and Kpn I; M, DNA marker; TFF3, trefoil factor 3.

the process of reconstitution through cell migration (7). The interaction between TFF3 and mucins has also been reported to protect intestinal epithelia from injuries, maintaining mucosal barrier function (26). Furthermore, mitogenic effects of TFF3 have been identified in vitro in primary cell culture, and TFF3 has been demonstrated to inhibit apoptosis, induce cellular invasion, act as an inflammatory modulator and exhibit pro-angiogenic activity (27-32). A protective or healing effect of TFF3 has also been reported in a series of experiments, such as cells in vitro, mice, rats and human (12-17). In addition, a previous study in TFF3-deficient mice demonstrated that the intestinal mucosa was damaged and apoptosis increased in the colon of the model mice compared with TFF3-competent controls (6). 


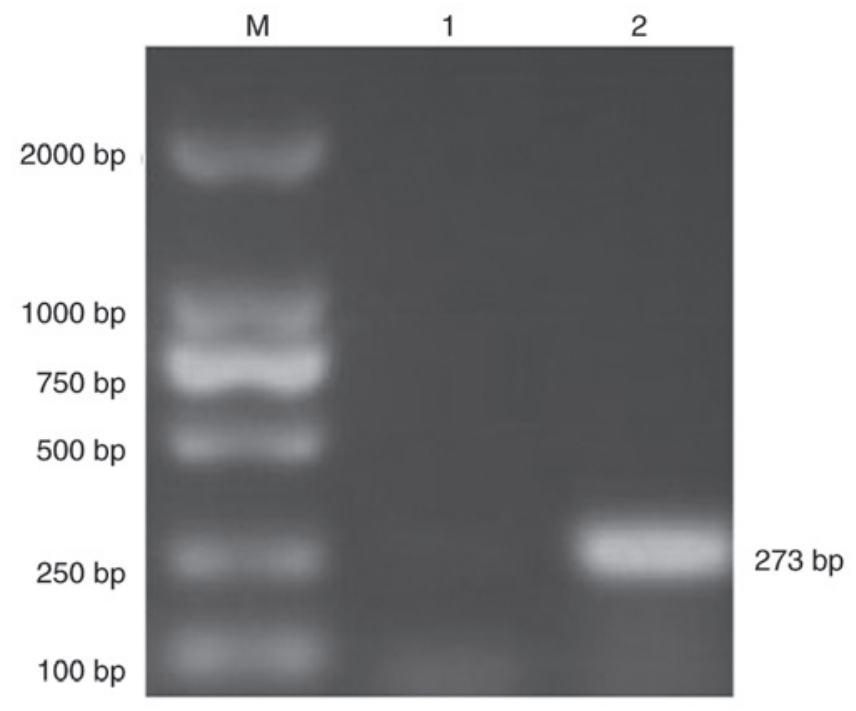

Figure 3. Electrophoretic analysis of amplified products for the TFF3-6xhis gene. Lane 1, negative control, the PCR product without template DNA; lane 2, PCR products with pNCMO2-TFF3-6xhis vector; M, DNA marker; TFF3, trefoil factor 3 .

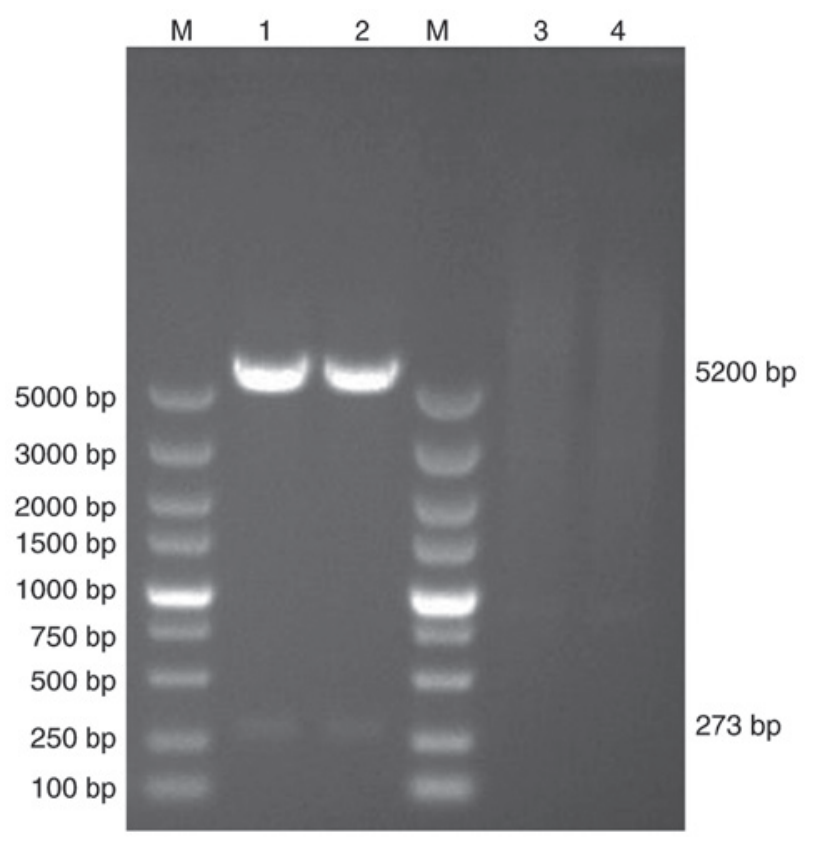

Figure 4. Identification of double enzyme digestion of the pNCMO2-TFF36xhis plasmid. Lane 1-2, pNCMO2-TFF3-6xhis plasmid digested by restriction enzymes Sal I and Kpn I; lane 3-4, double digestion without plasmid; M, DNA marker; TFF3, trefoil factor 3.

TFF3 is mainly secreted by the small intestine and colon goblet cells. However, it is very difficult to directly isolate natural TFF3 from these tissues (20-22). Thus, it is important to use genetic engineering strategies to produce large amounts of recombinant TFF3 for biochemical and biomedical applications. B. choshinensis has an exceptional capacity to produce heterologous proteins $(23,24,33-35)$. Target proteins can be produced and secreted efficiently with a high yield by Brevibacillus expression systems (33). The Brevibacillus expression system is suitable for producing eukaryotic proteins $(23,24,33,34)$. Compared with traditional

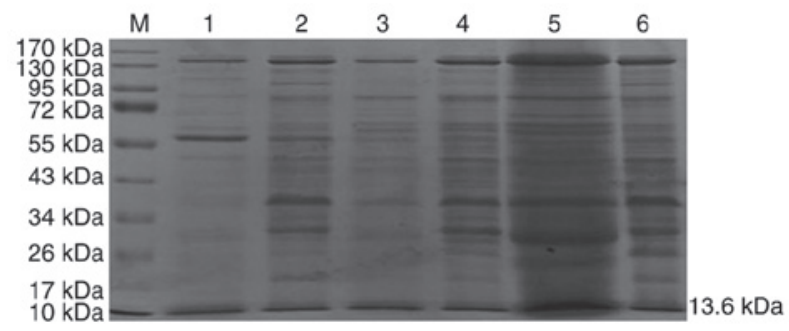

Figure 5. SDS-PAGE of Brevibacillus choshinensis HB116 transformants with pNCMO2 and pNCMO2-TFF3-6xhis plasmid. Lane 1, supernatant of the lysate of the position control vector-amylase after induction; lane 2 , precipitate of the lysate of the position control vector-amylase after induction; lane 3, supernatant of the lysate of the $\mathrm{pNCMO} 2$ vector after induction; lane 4, precipitate of the lysate of the pNCMO2 vector after induction; lane 5, supernatant of the lysate of the pNCMO2-TFF3-6xhis vector after induction; lane 6: precipitate of the lysate of the pNCMO2-TFF3-6xhis vector after induction; M, protein marker; TFF3, trefoil factor 3.

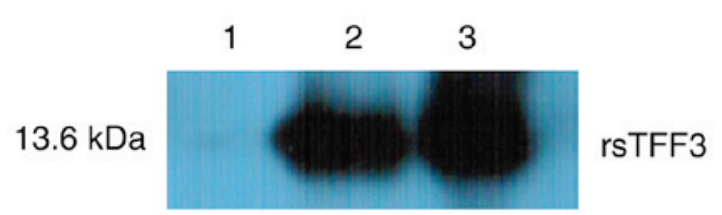

Figure 6. Western blot analysis of TFF3-6xhis fused protein. Lane 1, negative control, induction of vector-free bacteria; lanes 2 and 3 , supernatant protein of bacteria transfected with pNCMO2-TFF3-6xhis plasmid after induction. rsTFF3, recombinant secreted trefoil factor 3 .

E. coli expression systems, the genus Brevibacillus, including thermophilic, alkalophilic, psychrophilic, acidophilic and halophilic strains, use more carbon for heterotrophic or autotrophic growth (34). Brevibacillus expression systems can produce certain secretory or cytoplasmic proteins that $E$. coli expression systems fail to produce (35). Brevibacillus expression systems possess a good protein folding environment, lack proteases and have a convenient downstream processing model, e.g. cell separation and purification of secreted proteins from the culture medium (36). Thus a number of genetically engineered enzymes and heterologous proteins including cytokines, antigens and antibody fragments are expressed using Brevibacillus systems (33). Extracellular proteins, including several bacterial and mammalian proteins (with yields ranging between $10-1,250 \mathrm{mg} / \mathrm{l}$ ) have been produced using Brevibacillus systems (37-41). Recombinant TFF1 was secreted extracellularly with a high yield by the Brevibacillus system and had better wound healing capability compared with TFF1 produced by E. coli (37).

In the present study, the sus scrofa TFF3 gene was cloned into the pNCMO2 shuttle vector. $B$. choshinensis was used as a host bacterium to express sus scrofa TFF3 and produced $30 \mathrm{mg} / 1$ protein fused with a $6 x$ his-tag. Protein production was confirmed by SDS-PAGE and western blot analyses. The obtained fusion protein exhibited good antigenicity and specificity. Thus, Brevibacillus may be used to produce useful mucosal factors, which can be analyzed in terms of protein structure, bioactivity and kinetics as well as for their mucosal-protection activities. This expression system may be used in industrial applications to produce novel inhibitors of diarrhea. 


\section{Acknowledgements}

Not applicable.

\section{Funding}

This work was supported by grants from the National Key Research and Development Program of China (grant no. 2017YFD0501100) and the Colleges and Universities Key Research Plans in Henan (grant nos. 18A230009 and 19B230013).

\section{Availability of data and materials}

The datasets used and/or analyzed during the present study are available from the corresponding author on reasonable request.

\section{Authors' contributions}

YYW, HPL, CMX, BYW and KZ designed the study. YYW, HPL, CMX, BYW, KZ, YZZ, LPF and YDC performed the experiments. GMZ, XYJ, GYY and AQL analyzed the data. HPL, KZ and YYW wrote the manuscript. AQL drafted and revised the manuscript. All authors read and approved the final manuscript.

\section{Ethics approval and consent to participate}

Not applicable.

\section{Patient consent for publication}

Not applicable.

\section{Competing interests}

The authors declare that they have no competing interests.

\section{References}

1. Kjellev S: The trefoil factor family-small peptides with multiple functionalities. Cell Mol Life Sci 66: 1350-1369, 2009.

2. Aihara E, Engevik KA and Montrose MH: Trefoil factor peptides and gastrointestinal function. Annu Rev Physiol 79: 357-380, 2017

3. Sun Z, Liu H, Yang Z, Shao D, Zhang W, Ren Y, Sun B, Lin J, $\mathrm{Xu} \mathrm{M}$ and Nie S: Intestinal trefoil factor activates the PI3K/Akt signaling pathway to protect gastric mucosal epithelium from damage. Int J Oncol 45: 1123-1132, 2014.

4. Huynh E and Li J: Generation of lactococcus lactis capable of coexpressing epidermal growth factor and trefoil factor to enhance in vitro wound healing. Appl Microbiol Biotechnol 99: 4667-4677, 2015.

5. Fabisiak A, Bartoszek A, Kardas G, Fabisiak N and Fichna J: Possible application of trefoil factor family peptides in gastroesophageal reflux and Barrett's esophagus. Peptides 115: 27-31, 2019

6. Jeffrey GP, Oates PS, Wang TC, Babyatsky MW and Brand SJ: Spasmolytic polypeptide: A trefoil peptide secreted by rat gastric mucous cells. Gastroenterology 106: 336-345, 1994.

7. Lin N, Xu LF and Sun M: The protective effect of trefoil factor 3 on the intestinal tight junction barrier is mediated by toll-like receptor 2 via a PI3K/Akt dependent mechanism. Biochem Bioph Res Commun 440: 143-149, 2013.

8. Scholven J, Taras D, Sharbati S, Schon J, Gabler C, Huber O, Meyer zum Büschenfelde D, Blin N and Einspanier R: Intestinal expression of TFF and related genes during postnatal development in a piglet probiotic trial. Cell Physiol Biochem 23: 143-156, 2009.
9. Graziani F, Pinton P, Olleik H, Pujol A, Nicoletti C, Sicre M, Quinson N, Ajandouz EH, Perrier J, Pasquale ED, et al: Deoxynivalenol inhibits the expression of trefoil factors (TFF) by intestinal human and porcine goblet cells. Arch Toxicol 93: 1039-1049, 2019

10. Heo JM, Opapeju FO, Pluske JR, Kim JC, Hampson DJ and Nyachoti CM: Gastrointestinal health and function in weaned pigs: A review of feeding strategies to control post-weaning diarrhoea without using in-feed antimicrobial compounds. J Anim Physiol an N 97: 207-237, 2013.

11. Pluske JR, Turpin DL and Kim JC: Gastrointestinal tract (gut) health in the young pig. Anim Nutr 4: 187-196, 2018

12. Meyer zum Buschenfelde D, Hoschutzky H, Tauber R and Huber O: Molecular mechanisms involved in TFF3 peptide-mediated modulation of the E-cadherin/catenin cell adhesion complex. Peptides 25: 873-883, 2004.

13. Durer U, Hartig R, Bang S, Thim L and Hoffmann W: TFF3 and EGF induce different migration patterns of intestinal epithelial cells in vitro and trigger increased internalization of E-cadherin. Cell Physiol Biochem 20: 329-346, 2007.

14. Paulsen FP, Woon CW, Varoga D, Jansen A, Garreis F, Jager K, Amm M, Podolsky DK, Steven P, Barker NP and Sel S: Intestinal trefoil factor/TFF3 promotes re-epithelialization of corneal wounds. J Biol Chem 283: 13418-13427, 2008.

15. Jiang GX, Zhong XY, Cui YF, Liu W, Tai S, Wang ZD, Shi YG, Zhao SY and Li CL: IL-6/STAT3/TFF3 signaling regulates human biliary epithelial cell migration and wound healing in vitro. Mol Biol Rep 37: 3813-3818, 2010.

16. Manko A, Motta JP, Cotton JA, Feener T, Oyeyemi A, Vallance BA, Wallace JL and Buret AG: Giardia co-infection promotes the secretion of antimicrobial peptides beta-defensin 2 and trefoil factor 3 and attenuates attaching and effacing bacteria-induced intestinal disease. PLoS One 12: e0178647, 2017.

17. Nakov R, Velikova T, Nakov V, Ianiro G, Gerova V and Tankova L: Serum trefoil factor 3 predicts disease activity in patients with ulcerative colitis. Eur Rev Med Pharmacol Sci 23: 788-794, 2019.

18. Soriano-Izquierdo A, Gironella M, Massaguer A, May FE, Salas A, Sans M, Poulsom R, Thim L, Pique JM and Panes J: Trefoil peptide TFF2 treatment reduces VCAM-1 expression and leukocyte recruitment in experimental intestinal inflammation. J Leukoc Biol 75: 214-223, 2004.

19. Lin J, Holzman IR, Jiang P and Babyatsky MW: Expression of intestinal trefoil factor in developing rat intestine. Biol Neonate 76: 92-97, 1999.

20. Fang M, Wang W, Wang Y and Ru B: Bacterial expression and purification of biologically active human TFF3. Peptides 25: 785-792, 2004

21. Lu R, Wang X, Chen JP, Chen $X$ and Xu JN: Expression of human TFF3 in Escherichia coli. Sichuan Da Xue Xue Bao Yi Xue Ban 41: 114-117, 2010 (In Chinese).

22. Wang H, Tong Y, Fang M and Ru B: High-level expression of human TFF3 in Escherichia coli. Peptides 26: 1213-1218, 2005.

23. Mizukami M, Hanagata $\mathrm{H}$ and Miyauchi A: Brevibacillus expression system: Host-vector system for efficient production of secretory proteins. Curr Pharm Biotechnol 11: 251-258, 2010 .

24. Tokunaga M, Mizukami M, Yamasaki K, Tokunaga $\mathrm{H}$, Onishi H, Hanagata H, Ishibashi M, Miyauchi A, Tsumoto K and Arakawa T: Secretory production of single-chain antibody $(\mathrm{scFv})$ in Brevibacillus choshinensis using novel fusion partner. Appl Microbiol Biot 97: 8569-8580, 2013.

25. Uenishi H, Eguchi T, Suzuki K, Sawazaki T, Toki D, Shinkai H, Okumura N, Hamasima N and Awata T: PEDE (Pig EST Data Explorer): Construction of a database for ESTs derived from porcine full-length cDNA libraries. Nucleic Acids Res 1: D484-D488, 2004.

26. Kondo S, Araki T, Toiyama Y, Tanaka K, Kawamura M, Okugawa Y, Okita Y, Saigusa S, Inoue Y, Uchida K, et al: Downregulation of trefoil factor-3 expression in the rectum is associated with the development of ulcerative colitis-associated cancer. Oncol Lett 16: 3658-3664, 2018.

27. Gao F, Pan SX, Liu B and Zhang HZ: TFF3 knockout in human pituitary adenoma cell HP75 facilitates cell apoptosis via mitochondrial pathway. Int J Clin Exp Patho 8: 14568-14573, 2015.

28. Arnold P, Rickert U, Helmers AK, Spreu J, Schneppenheim J and Lucius R: Trefoil factor 3 shows anti-inflammatory effects on activated microglia. Cell Tissue Res 365: 3-11, 2016. 
29. Große-Kreul J, Busch M, Winter C, Pikos S, Stephan H and Dunker N: Forced trefoil factor family peptide 3 (TFF3) expression reduces growth, viability, and tumorigenicity of human retinoblastoma cell lines. PLoS One 11: e0163025, 2016.

30. Bastholm SK, Samson MH, Becher N, Hansen LK, Stubbe PR Chronakis IS, Nexo E and Uldbjerg N: Trefoil factor peptide 3 is positively correlated with the viscoelastic properties of the cervical mucus plug. Acta Obstet Gyn Scan 96: 47-52, 2017.

31. Li Q, Wang KK, Su C and Fang JY: Serum trefoil factor 3 as a protein biomarker for the diagnosis of colorectal cancer. Technol Cancer Res T 16: 440-445, 2017.

32. Al-Salam S, Sudhadevi M, Awwad A and Al Bashir M: Trefoil factors peptide-3 is associated with residual invasive breast carcinoma following neoadjuvant chemotherapy. Bmc Cancer 19: $135,2019$.

33. Mizukami M, Onishi $\mathrm{H}$, Hanagata $\mathrm{H}$, Miyauchi A, Ito $\mathrm{Y}$, Tokunaga $\mathrm{H}$, Ishibashi $\mathrm{M}$, Arakawa $\mathrm{T}$ and Tokunaga $\mathrm{M}$ : Efficient production of Trastuzumab Fab antibody fragments in Brevibacillus choshinensis expression system. Protein Expres Purif 150: 109-118, 2018.

34. Mizukami M, Tokunaga $\mathrm{H}$, Onishi $\mathrm{H}$, Ueno $\mathrm{Y}$, Hanagata $\mathrm{H}$, Miyazaki N, Kiyose N, Ito Y, Ishibashi M, Hagihara Y, et al: Highly efficient production of $\mathrm{VHH}$ antibody fragments in Brevibacillus choshinensis expression system. Protein Expres Purif 105: 23-32, 2015.

35. Hu W, Xiang JY, Kong P, Liu L, Xie QH and Xiang HY: Expression and characterization of a single-chain variable fragment against human LOX-1 in Escherichia coli and Brevibacillus choshinensis. J Microbiol Biotechn 27: 965-974, 2017.
36. Zou C, Duan XG and Wu J: Efficient extracellular expression of Bacillus deramificans pullulanase in Brevibacillus choshinensis. J Ind Microbiol Biot 43: 495-504, 2016.

37. Cheng YM, Lu MT and Yeh CM: Functional expression of recombinant human trefoil factor 1 by Escherichia coli and Brevibacillus choshinensis. Bmc Biotechnol 15: 32, 2015.

38. Hanagata $\mathrm{H}$ and Mizukami M: Production of recombinant $\mathrm{CCN}$ proteins by Brevibacillus choshinensis. Methods Mol Biol 1489: 85-93, 2017.

39. Li Z, Su L, Duan X, Wu D and Wu J: Efficient expression of maltohexaose-forming $\alpha$-amylase from Bacillus stearothermophilus in Brevibacillus choshinensis SP3 and its use in maltose production. Biomed Res Int 2017: 5479762, 2017.

40. Panfertsev EA, Baranova EV, Mochalov VV, Soloviev PV, Gorbatov AA and Biketov SF: Construction of recombinant strain Brevibacillus choshinensis for chimeric borrelia Dbpa antigen production. Klin Lab Diagn 63: 450-454, 2018 (In Russian).

41. Duan X, Shen Z, Zhang X, Wang Y and Huang Y: Production of recombinant beta-amylase of Bacillus aryabhattai. Prep Biochem Biotech 49: 88-94, 2019.

(i) (9) This work is licensed under a Creative Commons Attribution-NonCommercial-NoDerivatives 4.0 International (CC BY-NC-ND 4.0) License. 